\title{
Dysphonia in opera singers due to psychogenic disorders
}

\author{
Clarós $\mathrm{P}^{1 *}$, Karlikowska $\mathrm{A}^{1,2 *}$, Clarós-Pujol $\mathrm{A}^{1}$, Clarós $\mathrm{A}^{\mathbf{1}}$ and Pujol $\mathrm{C}^{1}$ \\ ${ }^{1}$ Clarós Clinic, c./Vergós 31, 08017 Barcelona, Spain \\ ${ }^{2}$ Scholarship Clarós Clinic, Kracow, Poland
}

\begin{abstract}
The point of this article is to make a diagnosis of psychological voice disorders easier by reviewing germane to the subject literature. Current view on terminology, classification, clinical manifestation and underlying psychological background of this rare condition is given. Secondly our aim is to asses prevalence ratio of psychological voice disorders in a group of 1520 professional opera singers - people with the most challenging voice effort among professional voice users. Our findings contradict common belief of high occurrence rate of this disorder among opera singers. Characteristics of this professional group are discussed, and a short example case report is described. Based on literature findings and authors personal experience we propose an outline of an assessment protocol when psychological voice disorder is suspected. The importance of multidisciplinary diagnosis involving laryngologist, psychologist, speech therapist and possibly a psychiatrist is pointed out.
\end{abstract}

\section{Introduction}

A voice disorder is diagnosed when quality of voice, loudness or pitch of sound is insufficient for communication or inappropriate for person's age, gender or cultural background [1].

According to American Speech-Language-Hearing Association [ASHA] voice disorder is also present when the patient is having concerns about his voice even if others do not perceive it as abnormal [2].

Organic speech or voice disorder has structural or neurological components that cause the speech disturbance (e.g. vocal nodules, polyps, hematoma of vocal folds, structural changes in the larynx due to aging, vocal tremor, spasmodic dysphonia, or paralysis of vocal folds, among others).

On the contrary, a functional speech disorder is a voice impairment that is caused by underlying psychological process with no organic pathology (or a non-severe one which doesn't justify the intensity of the symptoms) of the vocal folds not associated with neurological illness, or if the vocal folds lesions are secondary to the functional disorder.

This group could be divided into two subgroups of pathologies: muscle tension voice disorder (MVD) and psychogenic voice disorder (PVD) [3].

Muscle tension voice disorder (also called hyperfunctional voice disorder) is a phonation impairment which develops over time due to psychological process which causes disbalance of phonatory muscles. Examination reveals: excessively effortful, forced voice during all types of sound, as well as rare phonatory breaks and involvement of the false vocal folds which can in extreme cases lead to ventricular phonation.

There are two subtypes of MTVD: with no pathology to the vocal folds (MTVD1) or with secondary, developed overtime organic changes (MTVD2) such as vocal nodules, contact ulcers or polyps due to muscle hyperactivity.

This article focus is on the psychogenic voice disorders group.
Psychogenic voice disorder (PVD) is a voice impairment (foremost dysphonia or aphonia) caused by pathological psychological process with absence of organic structural or neurological pathologies. Its main characteristics include existence of symptom incongruity as the patient would manifest severe dysphonia but preserve non-verbal sounds of vegetative behaviors, such as coughing, laughing, or crying with normal glottic closure and reversibility of the voice pathology [4].

We present review of literature alongside with an interesting case of psychogenic dysphonia in opera singer.

\section{Different clinical presentations of PVD}

Presentations of psychogenic voice disorders may include different symptoms and with myriad of underlying psychological disorders causing them they form a very heterogeneous group.

One of the most common manifestations of PVD is dysphonia or aphonia. The disorder can be explained that an individual who doesn't have enough ways of coping with stress or difficult life situations may lose voluntary voice control because of intrinsic and extrinsic laryngeal muscles imbalance.

Recent belief is that this disequilibrium could be caused by either a conversion reaction where psychological problem is 'converted' into a somatic manifestation, or high level of emotional distress due to difficult life events or psychological conflicts for example over fear of expressing negative emotions [5].

In psychogenic dysphonia, voice quality is deteriorated, voice may be rough, breathy, asthenic, low or high pitched and hoarse or strained,

*Correspondence to: Pedro Clarós, Clarós Clinic, c./Vergós 31, 08017 Barcelona, Spain, Tel: +34932031212,+34639339252; E-mail: clinica@clinicaclaros.com

Key words: psychological voice disorders, opera singers, psychogenic dysphonia, psychogenic aphonia, multidisciplinary diagnosis

Received: February 05, 2019; Accepted: February 21, 2019; Published: February 25,2019 
less commonly diplophonia may be present (two different tones). Aphonia usually presents itself as an asthenic or normal whisper or rarely as a total inability to produce sound [6]. Patient usually preserves ability of normal glottic closure during cough. Individual may also present normal or better phonation accidentally, involuntarily when he or she doesn't realize it. Like mentioned before this may occur during coughing or making other vocal, non-verbal sounds for example crying or even gurgling water [7]. Patient may also find counting or singing easier than conducting a conversation.

The course of PVD can be highly variable, usually the onset is sudden, but it can also develop gradually over hours or days. In this type of disorder episodes of normal voice can occur intermittent with dysphonia depending on the patient's emotional attitude [8].

On examination of the larynx by an ENT (with video-laryngoscopy or laryngo-stroboscopy) PVD may present itself as anterior or posterior constriction of the aryepiglottic folds and/ or hyper or hypoactivity of vocal folds. Also, paradoxical vocal folds dysfunction can occur or false vocal folds (vestibular vocal folds) overactivity which in extreme cases may lead to ventricular phonation. Muscle misuse may lead to quick vocal fatigue and weak voice [1]. Patient often presents himself as very anxious, agitated with untypical facial expression, excessive face movements. Typically, he or she may be experiencing increased stress level or suppressed anger, sometimes poor sex identification or suffer from dysthymia/depression [9]. Other associated somatoform pathologies can be: habitual cough or hyperventilation syndrome. Globus sensation is very common and can be reported by the patient as inability of swallowing, foreign object sensation or even dyspnea. The diagnosis should be confirmed by psychological and speech therapy assessments. Occasionally PVD may resolve spontaneously, it more commonly remains for longer periods, sometimes for months, or even for years. In most cases it is alleviated with one voice therapy session; however, it is important to include longer psychological treatment. Even after successful therapy reoccurrences are common and may appear couple of times in the future [10].

Other type of PVD is puberphonia or mutational falsetto which can appear either in an adult male or adolescent boy for an unusually long period of time. In this condition the voice characteristics are: highpitched (usually a full octave or more above the normal pitch) asthenic, breathy, falsetto voice which can be intermittent by a normal voice [1].

Some authors believe that other rare manifestations may be a foreign accent syndrome which means developing foreign speech prosody without actually changing geographical surroundings; and childish voice in adults, where voice is usually of higher pitch, and childlike pattern, with no other than psychological reasons [11].

Mutism or selective mutism disorder is characterized by the fact that the patient usually just mouths words without making attempt of using voice when patient with elective mutism consciously chooses not to speak in some domains but can normally speak in others [12].

Another type of pathology, marked out only by some of the authors, is psychogenic adductor spasmodic dysphonia (ADSD). Voice presentation resembles neurological ADSA and could be strained or asthenic, with frequent or irregular phonatory breaks, sometimes a voice tremor may be present [1].

\section{Most commonly found psychological traits}

Studies about psychogenic implication in the pathogenesis of psychogenic voice disorders reveal significant depressive symptoms, elevated stress level, anxiety and adjustment disorders as well as eating disorders, hypochondriasis or hysteria.

In his study Willinger et al. [13] tested severity of depression and anxiety in 61 patients with functional dysphonia, and they concluded that as much as $33 \%$ of the patients showed mood disorder symptoms and $20 \%$ of them were overly anxious compared to the non-dysphonic control group matched by age, sex and occupation. This study also showed increased concern regarding health issues than other reasons for anxiety. Interesting is the fact that those symptoms seem to worsen after appearance of voice disorder, therefore we could argue what is the cause and what is the effect.

The same author conducted a study where he assessed personality aspects as: novelty seeking, harm avoidance, reward dependence and persistence (based on Cloninger's personality model) and their connection to psychological voice disorders. The results showed that harm avoidance scores were significantly elevated in comparison to control group but no meaningful differences in novelty seeking, reward dependence or persistence appeared [14].

Different comparative study made by Kotby et al. [15] reviewed degree of severity of anxiety symptoms in 100 patients diagnosed with functional voice disorders and 50 normal individuals (control group). The result in patients with functional disorders reached $43 \%$ of individuals but only $6 \%$ had elevated anxiety level in healthy control group. It also concluded that other often associated psychological traits are hypochondriasis (especially in puberphonia), somatization, hysteria, distress, and psychogenic pain disorder.

Martins and al. [16] give some important clinical characteristics in his case series report. His findings included profession (the most common was a housekeeper) type of onset (most common was sudden onset) and the most common manifestation (which was conversion aphonia alongside with muscle tension disorder and intermittent voicing). The prevalence rate of women was $26: 2$ in a not a very numerous groups of 28 patients. Tezcanez et al. [17] when examined the factor of patient's employment found the most common was also housewife $31 \%$ before retired $18 \%$.

On the other hand, Reiter et al. [18] assessed that the most common profession to develop this malady are business related, highly stressful positions like businessman/ businesswoman.

Another interesting study about prevalence of orthorexia nervosa among the performance artists (in the State Opera and Ballet and in the Bilkent University Symphony Orchestra) was submitted by Aksoydan et al. [19]. This study showed increased frequency in artists of orthorexia than control group with highest prevalence of eating disorders recorded among opera singers (81.8\%).

Etiology of this disorder was described by Andersson et al. [20] She examined the most common type of underlying reasons to this pathology. Her findings revealed that interpersonal conflicts related firstly to family and secondly to work are the most common causes of distress. She also believes that cognitive and behavioral therapy alone is not enough for PVD patients as it should be accompanied by voice therapy and therapeutic counselling.

The therapeutic approach has changed a lot during years. The outdated methods were quite violent and aimed at regaining voice at first voice therapy session. Worth mentioning are historic methods like obstructing the larynx until patient would feel a need to scream, applying electrical impulses or irritating the larynx by powder or fluid or cocaine solution [21]. 
Big longitudinal study on a group of 500 patients took place between year 1972 and 2004. Patients were treated for psychogenic aphonia with more suitable methods of treatment: respiration, relaxation and phonetic exercise, inhalation phonation, gargle and chewing. However, in the study authors strongly point out the importance of quick resolution of symptoms preferably during the first voice therapy session [22], which seems not to be accurate. At present recommended approach is psychodynamic-systemic therapy helping the patient understand the essence of the underlying problem. It is no longer believed that aphonia should be alleviated as soon as possible but rather in the patient's own time [21].

Sixty-eight patients took part in an uncontrolled study conducted by Martinez et al. [23] Its aim was to determine how voice therapy affects not only voice parameters but also what is the change in anxiety and depression symptoms before and after voice therapy. The result was that not only it improves quality of the voice but also alleviates (in patients self-evaluation) symptoms of anxiety and depression.

\section{How frequent is PVD in singers?}

The data from literature put prevalence rate of all voice disorders in general adult population between $6,6 \%$ at the time of the study [24] to $7,6 \%$ in the last 12 months [25] It is believed that voice disorder's lifetime occurrence rate oscillates around $29,1 \%$ [26] Some researchers estimate that the prevalence rate of PVD is $0,4 \%$ in general population ${ }^{21}$ which corresponds to reports where $10-40 \%$ of all voice disorders have some kind of underlying or coexisting functional cause [27]. Psychological voice disorders are more often in women with a predominance varying between different studies. Baker et al estimated that ratio at $8: 1$ by reviewing associated literature [28]. It was also conducted that the period of life with the highest professional voice activity (30-50 years old) would be the most endangered with developing this illness [24].

Singers were reported $2.43 \%$ among all voice patients in a study of occupational risks conducted by Verdolini et al. [29], but Titze et al. [30] found that $11.5 \%$ of the clinical voice population was composed of singers, and there was a high representation of non-classical singers.

In other study, Pestana et al. [31] conducted a meta-analysis of the prevalence of self-reported voice disorders in singers. The result went as high as $46 \%$, far superior to $18.8 \%$ in control group. Like the author says it can be suspected that singers have more concerns about their voice than the rest of the population, still the result is surprisingly high.

Within professional voice users, opera singers are concerned the ones with the most challenging use of voice. Furthermore, the stress level in this group is estimated high above average. The common belief is that they are more susceptible to voice disorders. However, our findings contradict this idea.

\section{Personal experience with opera singers}

Clarós Clinic has been the reference center for voice care for many opera singers of several opera houses all over the world since more than 40 years. One of them is the Barcelona's opera house, the Gran Teatre del Liceu. During that time more than 1520 singers were treated in our clinic due to different voice impairments both organic and functional. Within this large group only 34 singers were, to our knowledge, diagnosed with a psychogenic voice disorder: 26 with psychogenic dysphonia (with various types of voice impairment) and 8 with psychogenic aphonia.

Among these 34 patients: 20 were sopranos (58,8\%) 5 were mezzosopranos $(14,8 \%), 3$ were countertenors $(8,8 \%)$, 3 were tenors $(8,8 \%)$,
2 were baritones (5,9\%) and 1 was contralto (2,9\%). No bass singer was diagnosed with this disorder.

Number of women was 26 singers $(76,5 \%)$ and there were 8 male singers $(23,5 \%)$. From this data it can be concluded that female to male prevalence ratio was $3,25: 1$ and that prevalence rate of psychogenic voice disorder, in the group of opera singers with all the voice disorders, over the last 40 years was 2,2\%. Also, more susceptible to developing PVD are singers with high tessituras than with low.

There are no medical records to report vocal fatigue, alcohol, drugs or tobacco use in any of the 34 singers. During their examinations, no substantial organic vocal pathology was discovered and neither were any symptoms of the inflammatory disease of the larynx.

Patients did not use routinely any medications, which may alter voice emission, for example, medication for thyroid function, hormones, and hypertension or diabetes medicaments. No history of relevant neurological disease (for example Parkinson disease or myasthenia gravis) was reported.

However, there are data that 24 of the 34 were taking antipsychotic drugs to treat the anxiety, in a routine way (Diazepam, Fluoxetine, Sertraline, Paroxetine, Citalopram, Escitalopram, Lorazepam, Alprazolam or Clonazepam.)

\section{Case report}

A 52 years old baritone opera singer, from Germany, expert on Wagner's operas, suddenly presented loss of power of his voice, just at the end of the second act of the performance. He was the lead male singer in Siegfried opera written by Wagner and played at the Opera House in Barcelona. During overture after the second act (which takes $15 \mathrm{~min}$ ) his symptoms intensified, and he presented with weak, strained and breathy voice. The famous opera singer was terrified and wanted to cancel the rest of his performance.

Medical examination of the larynx showed no organic lesions on his vocal folds, no secretion was present. The color of the mucosa was normal. Normal movement of vocal folds, their closure and phonatory function was correct. Basic test of coughing was performed-patients cough showed no pathological features.

On further examination slight muscular tenderness was present in the back of the neck and the perilaryngeal area.

Patient presented difficulty in communicating, spontaneous anomalous movements of the eye lids and lips, and autonomic symptoms of forehead sweating and face flushing. When asked, he admitted to having dry mouth and feeling weak. Time was running out, so the next question was if it ever had happened to him before. His answer was yes, 2 years before the incident. His personal situation at the time was difficult as he had been in the process of getting a divorce with his first wife at that time.

When asked about his present marital situation he admitted to having a crisis with his second wife and that the word "divorce" was used on that very day. After having realized that fact his voice improved and after a few voices and phonatory exercises he resumed with his professional activities and gave a good performance in the third act.

A provisional diagnosis of psychogenic voice disorder was made in the form of mild dysphonia. On the subsequent day he started psychological treatment. His evaluation confirmed the diagnosis. No previous psychiatric problems were referred before. Patient denied use of tobacco, alcohol, drugs or any new medication. His voice use (the 
number of hours a day practicing singing) was not significantly different from standard at the time. There were no changes in work conditions or significant deterioration of atmosphere in his work environment found.

More detailed history revealed that he was a son of a protestant priest who very strictly condemned his first divorce causing a lot of distress to the patient. His second wife was much younger than him and a very attractive woman and many differences occurred between them in everyday life.

His anxiety level was assessed using Hamilton Anxiety rating scale HAM-A with a score pointing to moderate/severe anxiety level. Alongside with the long-term psychological treatment he received help from the speech therapist and there were no recurrences so far.

His family life also improved, and he is a father of a one-year old girl at present. He modified his professional career and now he is running his master class sessions as a voice conduct teacher.

Interesting is also the fact that his dysphonia begun in the third scene of the second act of the Siegfried opera where a female character (Brunhilde) appears.

\section{Discussion}

Making a diagnosis of patient with a PVD is a difficult process, especially with professional singers. As shown in the example above sometimes it is essential to keep in mind other than organic causes of voice disorders. Authors would suggest that the diagnosis process should be conducted in the following way: every patient with a voice complaint should undergo a voice assessment protocol. Primarily she or he should be examined by an ENT specialist using 70- or 90-degree rigid endoscope or fiberscope with HD camera with the possibility of recording. Examination should be conducted during articulation of /i/a and singing of low and high-pitched sounds. As doctor performs videolaryngoscopy (VLS) or videofibrolaryngoscopy he can detect most organic causes of voice disorders.

Coexisting medical conditions should be diagnosed and treated (for example GERD or rhinosinusitis).

Next a videostroboscopy or high-speed digital imaging (HSDI) should be performed to asses if the mobility of the vocal cords is symmetric, their amplitudes and mucosal waves normal. Vocal folds closure should be complete and their movement regular. Videostroboscopy together with a manual examination of neck in the larynx area (which can reveal muscle tension and pain) allows determining and excluding MVD group of disorders. Those patients should be referred to speech therapist and closely monitored by an ENT specialist for any occurring vocal folds lesions.

The remaining group of patients is likely to have a psychogenic voice disorder. A simple test of cough can help detect the hysteric origin of the process. Before referring patient to psychologist, the speaking voice should be assessed in regard to grade of dysphonia (severity of the disorder) roughness, breathiness, asthenia, strain (GRBAS scale and/or VHI scale) Pitch and voice range should be defined (using a Bruel\&Kjaer stroboscope or High-Speed Digital Imaging -HSDI). High definition recordings of the voice should be made.

Breathing patterns should be assessed and if necessary improved by learning to activate and loosen muscles breathing to improve resonance of the voice.

Thorough psychological evaluation should be conducted, aimed to asses stress and anxiety levels using standardized scales (e.g. Hamilton
Anxiety rating scale HAM-A Hamilton Anxiety Rating Scale for Depression HAM-D, Beck depression Inventory BDI, State-Trait Anxiety Inventory STAI, Hospital Anxiety and Depression Scale HADS scale) and also psychoanalysis should define if any stressful life event occurred or if the patient is suffering from conversion reaction or any other psychological conflict (e.g. over speaking out). If necessary also a thorough psychiatric examination should be performed.

\section{Conclusion}

In contrast to the common belief prevalence rate of voice disorders in opera singers in not higher than other professional voice users: it was $2,2 \%$ in the group of 1520 opera singers over the last 40 years. In authors opinion they owe it to increased (compared to other people) voice care. It is important to point out that professional opera singers are a very specific group of people, very reluctant to give out any personal detail, especially regarding their voice disorders or difficulties in private life. For this reason, we believe, some pathologies may not be routinely communicated to the general practitioners.

Psychological voice disorder is a very rare and complicated illness which is often misdiagnosed and therefore inadequately treated. Both the diagnosis and the treatment of this condition should be multidisciplinary involving speech therapist, psychologist sometimes psychiatrist and a laryngologist, as each of the specialists has an important role in the process. Establishing diagnostic criteria not only for voice assessment but also for psychological diagnosis would help to improve the process. Making a correct diagnosis of PVD is a task for a group of specialists but also a disorder which mustn't be forgotten by general practitioners.

\section{References}

1. Baker J, Ben-Tovim DI, Butcher A, Esterman A, McLaughlin K (2007) Development of a modified diagnostic classification system for voice disorders with inter-rater reliability study. Logopedics Logoped Phoniatr Vocol 32: 99-112. [Crossref]

2. Andrea M, Andrea M, Figueira ML (2018) Self-perception of quality of life in patients with functional voice disorders: the effects of psychological and vocal acoustic variables. European Eur Arch Otorhinolaryngol 275: 2745-2754. [Crossref]

3. Ludlow CL, Domangue R, Sharma D, Jinnah HA, Perlmutter JS, et al. (2018) Consensus-Based Attributes for Identifying Patients with Spasmodic Dysphonia and Other Voice Disorders. JAMA Otolaryngol Head Neck Surg 144: 657. [Crossref]

4. Schalén L, Andersson K, Eliasson I (1992) Diagnosis of psychogenic dysphonia. Acta Otolaryngol Suppl 492: 110-112. [Crossref]

5. Baker J (2016) Functional voice disorders: clinical presentations and differential diagnosis. Handb Clin Neurol 139: 389-405. [Crossref]

6. Mathieson L (2013) Greene and Mathieson's the Voice and its Disorders. ( $6^{\text {th }}$ edn), John Wiley \& Sons: 750.

7. House A, Andrews HB (1987) The psychiatric and social characteristics of patients with functional dysphonia. $J$ Psychosom Res 31: 483-490. [Crossref]

8. Butcher P (1995) Psychological processes in psychogenic voice disorder. Int J Lang Commun Disord 30: 467-474.

9. Baker J (2008) The role of psychogenic and psychosocial factors in the development of functional voice disorders. Int J Speech Lang Pathol 10: 210-230. [Crossref]

10. Roy N, Bless DM (2000) Personality traits and psychological factors in voice pathology: a foundation for future research. J Speech Lang Hear Res 43: 737-748. [Crossref]

11. Keulen S, Verhoeven J, De Witte E, De Page L, Bastiaanse R, et al. (2016) Foreign accent syndrome as a psychogenic disorder: a review. Front Hum Neurosci 10: 168 [Crossref]

12. Rubin J (2016) The professional voice user. In Practical Laryngology CRC Press, Boca Raton, FL: 19-26.

13. Willinger U, Völkl-Kernstock S, Aschauer, HN (2005) Marked depression and anxiety in patients with functional dysphonia. Psychiatry Res 134: 85-91. [Crossref] 
14. Willinger U, Aschauer HN (2005) Personality, anxiety and functional dysphonia. Pers Individ Dif 39: 1441-1449.

15. Kotby MN, Baraka M, El Sady SR, Ghanem M, Shoeib R (2013) Psychogenic stress as a possible etiological factor in non-organic dysphonia. Int Congr Ser 1240: 1251-1256.

16. Martins RHG, Tavares ELM, Ranalli PF, Branco A, Pessin ABB (2014) Psychogenic dysphonia: Diversity of clinical and vocal manifestations in a case series. Braz J Otorhinolaryngol 80: 497-502. [Crossref]

17. Tezcaner ZC, Gökmen MF, Yildirim S, Dursun G (2017) Clinical Features of Psychogenic Voice Disorder and the Efficiency of Voice Therapy and Psychological Evaluation. $J$ Voice 33: 250-254. [Crossref]

18. Reiter R, Rommel D, Brosch S (2013) Long term outcome of psychogenic voice disorders. Auris Nasus Larynx 40: 470-475. [Crossref]

19. Aksoydan E, Camci N (2009) Prevalence of orthorexia nervosa among Turkish performance artists. Eat Weight Disord 14: 33-37. [Crossref]

20. Andersson K, Schalén L (1998) Etiology and treatment of psychogenic voice disorder: results of a follow-up study of thirty patients. $J$ Voice 12: 96-106. [Crossref]

21. Kolbrunner J, Menet AD, Seifert E (2010) Psychogenic aphonia: no fixation even after a lengthy period of aphonia. Swiss Med Wkly 140: 12-17. [Crossref]

22. Maniecka-Aleksandrowicz B, Domeracka-Kolodziej A, Rózak-Komorowska A Szeptycka-Adamus A (2006) Management and therapy in functional aphonia: analysis of 500 cases. Otolaryngol Pol 60: 191-197. [Crossref]
23. Martinez CC, Cassol M (2015) Measurement of voice quality, anxiety and depression symptoms after speech therapy. J Voice 29: 446-449. [Crossref]

24. Roy N, Merrill RM, Gray SD, Smith EM (2005) Voice disorders in the general population: prevalence, risk factors, and occupational impact. The Laryngoscope 115: 1988-1995. [Crossref]

25. Bhattacharyya $\mathrm{N}$ (2014) The prevalence of voice problems among adults in the United States. Laryngoscope 124: 2359-2362. [Crossref]

26. Cohen SM (2010) Self-reported impact of dysphonia in a primary care population: an epidemiological study. Laryngoscope 120: 2022-2032. [Crossref]

27. Andrea M, Andrea M, Figueira ML (2018) Self-perception of quality of life in patients with functional voice disorders: the effects of psychological and vocal acoustic variables. Eur Arch Otorhinolaryngol 275: 2745-2754. [Crossref]

28. Baker J (2002) Psychogenic voice disorders--heroes or hysterics? A brief overview with questions and discussion. Logoped Phoniatr Vocol 27: 84-91; discussion 91. [Crossref]

29. Verdolini K, Ramig LO (2001) Occupational risks for voice problems. Logoped Phoniatr Vocol 26: 37-46. [Crossref]

30. Titze IR, Lemke J, Montequin D (1997) Populations in the U.S. workforce who rely on voice as a primary tool of trade: a preliminary report. $J$ Voice 11: 254-259. [Crossref]

31. Pestana PM, Vaz-Freitas S, Manso MC (2017) Prevalence of voice disorders in singers: systematic review and meta-analysis. J Voice 31: 722-727. [Crossreff]

Copyright: @2019 Clarós P. This is an open-access article distributed under the terms of the Creative Commons Attribution License, which permits unrestricted use, distribution, and reproduction in any medium, provided the original author and source are credited. 